University of South Carolina

Scholar Commons

3-3-2004

\title{
Synthesis of Tin Oxide Nanostructures with Controlled Particle Size Using Mesoporous Frameworks
}

A. Cabot

Universitat de Barcelona

J. Arbiol

Universitat de Barcelona

E. Rossinyol

Universitat de Barcelona

J. R. Morante

Universitat de Barcelona

Fanglin Chen

University of South Carolina - Columbia, fchen@sc.edu

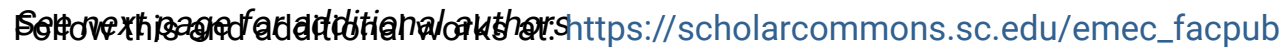

Part of the Mechanical Engineering Commons

\section{Publication Info}

Electrochemical and Solid-State Letters, Volume 7, Issue 5, 2004, pages G93-G97.

(c) The Electrochemical Society, Inc. 2004. All rights reserved. Except as provided under U.S. copyright law, this work may not be reproduced, resold, distributed, or modified without the express permission of The Electrochemical Society (ECS). The archival version of this work was published in Electrochemical and Solid-State Letters.

Publisher's Version: http://dx.doi.org/10.1149/1.1664054

PACS: 61.46.+w, 81.20.Ev, 82.45.Yz

This Article is brought to you by the Mechanical Engineering, Department of at Scholar Commons. It has been accepted for inclusion in Faculty Publications by an authorized administrator of Scholar Commons. For more information, please contact digres@mailbox.sc.edu. 


\section{Author(s)}

A. Cabot, J. Arbiol, E. Rossinyol, J. R. Morante, Fanglin Chen, and Meilin Liu

This article is available at Scholar Commons: https://scholarcommons.sc.edu/emec_facpub/3 


\title{
Synthesis of Tin Oxide Nanostructures with Controlled Particle
Size Using Mesoporous Frameworks
}

\author{
A. Cabot, ${ }^{\mathrm{a}, \mathrm{z}}$ J. Arbiol, ${ }^{\mathrm{a}}$ E. Rossinyol, ${ }^{\mathrm{a}}$ J. R. Morante, ${ }^{\mathrm{a}, *}$ Fanglin Chen, ${ }^{\mathrm{b}, *}$ \\ and Meilin Liu ${ }^{\mathrm{b}, *}$ \\ ${ }^{a}$ Enginyeria i Materials Electrònics, Department d'Electrònica, Universitat de Barcelona, \\ E-08028 Barcelona, Spain \\ ${ }^{b}$ School of Material Science and Engineering, Georgia Institute of Technology, Atlanta, \\ Georgia 30332-0245, USA
}

Tin oxide nanostructures with controlled narrow particle size distribution were synthesized inside silica mesoporous templates. In this way, particle growth was blocked by physically corseting the tin compound inside the silica frameworks, the pore diameter of which determines the final tin oxide crystallite size distribution. Template structures were subsequently eliminated by chemical methods to collect the unsupported semiconductor nanoparticles. Thus obtained tin oxide nanopowders, with particle sizes in the range between 6 and $10 \mathrm{~nm}$, were structurally, chemically, and electrically characterized. The results are compared with those obtained from the characterization of larger crystallite materials.

(C) 2004 The Electrochemical Society. [DOI: 10.1149/1.1664054] All rights reserved.

Manuscript submitted June 26, 2003; revised manuscript received September 9, 2003. Available electronically March 3 , 2004.

Nanoscience and nanotechnology are attracting increasingly more attention in recent years. In a parallel way, there is an urgent need to develop reliable methods to synthesize nanoparticles with a precisely controlled optical, magnetic, electric, or catalytic functionality, which strongly depends on particle size, shape, agglomeration, and surface states.

Tin oxide nanoparticles have attained much interest in the fields of optoelectronics, catalysis, and semiconductor gas sensors. They are usually obtained by sol-gel processes, although novel approaches have been successfully developed to synthesize large surface area materials. ${ }^{1}$

Sol-gel methods have shown success in obtaining very tiny nanoparticles when applying mild stabilization treatments. Nevertheless, for technological applications, stability, remaining residual elements, and semiamorphous surfaces are major problems to be avoided with more severe thermal treatments, which lead to large particle growth. Moreover, there is no control in morphology, and broad size distributions are usually obtained. Nanoparticle arrangement and agglomeration determine the final active surface area and are also a major factor for collective effects such as intergrain electrical conductance.

Synthesis of metal and semiconductor nanoparticles, ${ }^{2,3}$ nanotubules, ${ }^{4,5}$ and nanowires ${ }^{6}$ by encapsulation on template structures has been revealed recently as a promising method for obtaining small diameter monodispersed nanostructures. ${ }^{7}$ Attention has been mostly focused on supported metal aggregates for catalytic or optoelectronic applications. Template synthesized materials have particle size determined by pore diameter distributions of the framework structure. Further, the amount of precursor element deposited dictates the shape and size of the obtained nanostructures. ${ }^{7}$ In this way, monolayer, nanotubule, nanoparticles, or nanowires formation inside the channels can be controlled. ${ }^{4,8}$ Dispersion is accomplished by the isolation of the nanostructure into the template channels, thus enhancing its resistance to agglomeration. Nevertheless final agglomeration also depends on the template elimination procedure and posterior treatments. ${ }^{2}$

Here we report the template synthesis of tin oxide nanoparticles with controlled narrow grain size distributions. This is achieved by the nucleation and stabilization of the tin oxide while encapsulated in mesoporous silica frameworks. The template structures are subsequently eliminated by chemical methods to collect the unsupported semiconductor nanoparticles. Complete template elimination is required for electrical application of the nanostructures.

* Electrochemical Society Active Member.

z E-mail: acabot@el.ub.es

\section{Experimental}

SBA-15 mesoporous silica was used as a template for the synthesis of the tin oxide nanostructures due to its relatively large wall thickness and wide pore diameter window from 4 to $30 \mathrm{~nm},{ }^{9}$ thus allowing a relatively wide range of the final semiconductor nanoparticle size. SBA-15 mesoporous silicas were synthesized using pluronic P123 triblock copolymer and tetraethoxysilane, following the procedure reported by Zhao et al. ${ }^{9}$ Silica structures were calcined at $550^{\circ} \mathrm{C}$ for $4 \mathrm{~h}$. SBA- 15 frameworks were subsequently impregnated with an aqueous solution of tin oxalate with nitric acid. Proper selection of the initial precursor solution was critical to get $\mathrm{SnO}_{2}$ formed inside the pores. Incorporation of the tin nitrate compound on the mesoporous structure was achieved in five successive steps to avoid the formation of large tin oxide agglomerates outside the silica pores. The total amount of the tin compound precursor introduced was based on filling the pore volume of the silica structure, which was measured to be $1.1 \mathrm{~mL} / \mathrm{g}$. After impregnation, the material was dried in vacuum, to enhance tin composite diffusion inside the mesopores, and at room temperature to block premature tin oxide formation outside. Tin oxide was obtained by calcination of the tin nitrate inside the pores of SBA-15 at temperatures ranging from 500 to $800^{\circ} \mathrm{C}$ for $4 \mathrm{~h}$. After calcination, the template was eliminated by multiple washing steps with $1 \mathrm{M}$ sodium hydroxide solution in deionized water and ethanol at a 1:1 ratio. Once the silica template was removed, a second firing of the material, at $500^{\circ} \mathrm{C}$ was performed to ensure stabilization of the unsupported material and to eliminate residual impurities originating on the template dissolution.

For comparison, a commercial tin oxide powder (Alfa Aesar) with nonnanometric particle size was analyzed as well and is referred to here as the reference material. At the same time, $\mathrm{SnO}_{2}$ nanopowders obtained by precipitation of tin tetrachloride with an ammonia solution were characterized. The mean particle size of this material, which was calcined at $600^{\circ} \mathrm{C}$, is around $50 \mathrm{~nm}$.

Nitrogen adsorption/desorption isotherms at $77 \mathrm{~K}$ were obtained by a Coulter SA 3100 instrument. The surface area and pore-size distributions were calculated using Brunauer-Emmet-Teller (BET) and Barrett-Joyner-Halenda (BJH) methods, respectively. X-ray diffraction (XRD) spectra of the powders were recorded with a $\mathrm{Si}$ emens D500 X-ray diffractometer using $\mathrm{Cu} \mathrm{K} \alpha$ radiation. X-ray photoelectron spectroscopy (XPS) spectra were obtained on a Physical Electronics 5500 spectrometer, using monochromated Al Ka radiation. Tin oxide nanopowders were mounted to ensure an adequate electric contact with the holder. To minimize discrepancies due to the differences in the powder compactness, the amount of material, possible charging effects and contact resistance, all the measurement conditions and parameters were verified to get the $\mathrm{C} 1 \mathrm{~s}$ core level of the adventitious carbon at $284.8 \mathrm{eV}$. The Raman scattering measure- 
ments were obtained in backscattering geometry with a Jobin-Yvon T64000 spectrometer coupled to an Olympus metallographic microscope. Excitation was provided by an argon-ion laser operating at a wavelength of $488.0 \mathrm{~nm}$ with a low incident power to avoid thermal effects. Raman shifts were corrected using a silicon reference spectrum measured after each analysis of the $\mathrm{SnO}_{2}$ powders. Transmission electron microscope (TEM) measurements were done to characterize the template structures and extract the grain size distribution of the powders. High resolution TEM (HRTEM) measurements were carried out in a Philips CM30 SuperTwin electron microscope operating at $300 \mathrm{keV}$. For TEM examination, the powders were dispersed ultrasonically in ethanol and deposited on amorphous carbon membranes.

For the electrical characterization, sensors were fabricated by mixing the powders with an organic binder and then printing the obtained paste on alumina substrates having Pt electrodes and a heater. Resistance dependence as a function of the temperature was obtained by heating the sensors in different successive $20^{\circ} \mathrm{C}$ steps, from room temperature to $600^{\circ} \mathrm{C}$. The sensors were kept $15 \mathrm{~min}$ at each temperature, while resistance was monitored continuously. As a steady-state value, an average of the last few points was considered at each step. Prior to each measurement, sensors were stabilized at $550^{\circ} \mathrm{C}$ overnight.

\section{Results and Discussion}

Figure 1 shows TEM images of the hexagonal array of mesopores, which are arranged in structures grouping a few hundred pores with lengths up to $2 \mu \mathrm{m}$. The digital diffractogram (Fourier transform) analysis of the TEM images allows one to calculate a mesopore lattice constant of $10.2 \mathrm{~nm}$. The $\mathrm{N}_{2}$-sorption isotherm of the mesoporous silica clearly shows step and hysteresis loop corresponding to pore condensation. Pore volume distribution calculated using the BJH method was centered at a diameter of $7.2 \mathrm{~nm}$ (Fig. 2). Comparison of the structure periodicity with the pore size distribution allows one to calculate a wall thickness of $4.6 \mathrm{~nm} .{ }^{9}$

The BET surface area of the initial framework structure was 570 $\mathrm{m}^{2} / \mathrm{g}$. After filling the mesopores with tin oxide, the specific surface area decreases to $75 \mathrm{~m}^{2} / \mathrm{g}$. The BJH pore size distributions, calculated from the desorption branch, are plotted in Fig. 2. As expected, the total pore volume greatly decreases after filling the mesopores with tin oxide nanoparticles. The sorption isotherm of the tin oxide loaded silica has a double step associated with two pore size distributions. The first step is at the same relative pressure as that of the SBA-15. The second step is the only one present after dissolving off the silica template, this corresponds to some extent, to a mesoporic arrangement of the tin oxide particles, although more results are needed to clarify its origin.

Previous work on silica-supported elements reported a decrease of the pore diameter related to the template structure, which is associated with a recovery of the inner surface with the impregnated element, ${ }^{5}$ or to a shrinkage of the template due to a further condensation of the silicates with the incorporation process. ${ }^{2}$ However, in this study pore volume is nearly completely filled, therefore no decrease of the pore diameter is expected due to wall recovery. Moreover, TEM images reveal the conservation of the hexagonal mesoporous structure. This cannot be discerned in the XRD spectra of the tin compound loaded silica due to the decrease in electronic density contrast between the amorphous $\mathrm{SiO}_{2}$ walls and the tin oxide embedded between them. ${ }^{3,10}$ The preservation of SBA-15 structure is attributed to its wide wall thickness and the relatively high thermal treatments employed, and is crucial for a reliable control of the nanoparticle size distribution. However, a slight deformation of the channels is considered to occur with the highest calcination temperatures used here. This is the cause of the small increase of the mean nanoparticle size distribution observed. The fitting of the wide angle XRD peaks corresponding to the supported tin oxide crystallites, allows one to calculate (using Scherer's equation) grain sizes of $6.4,7.1$, and $8.4 \mathrm{~nm}$ after calcination at 500,600 , and $800^{\circ} \mathrm{C}$ for $4 \mathrm{~h}$, respectively. The initial silica template structures were calcined
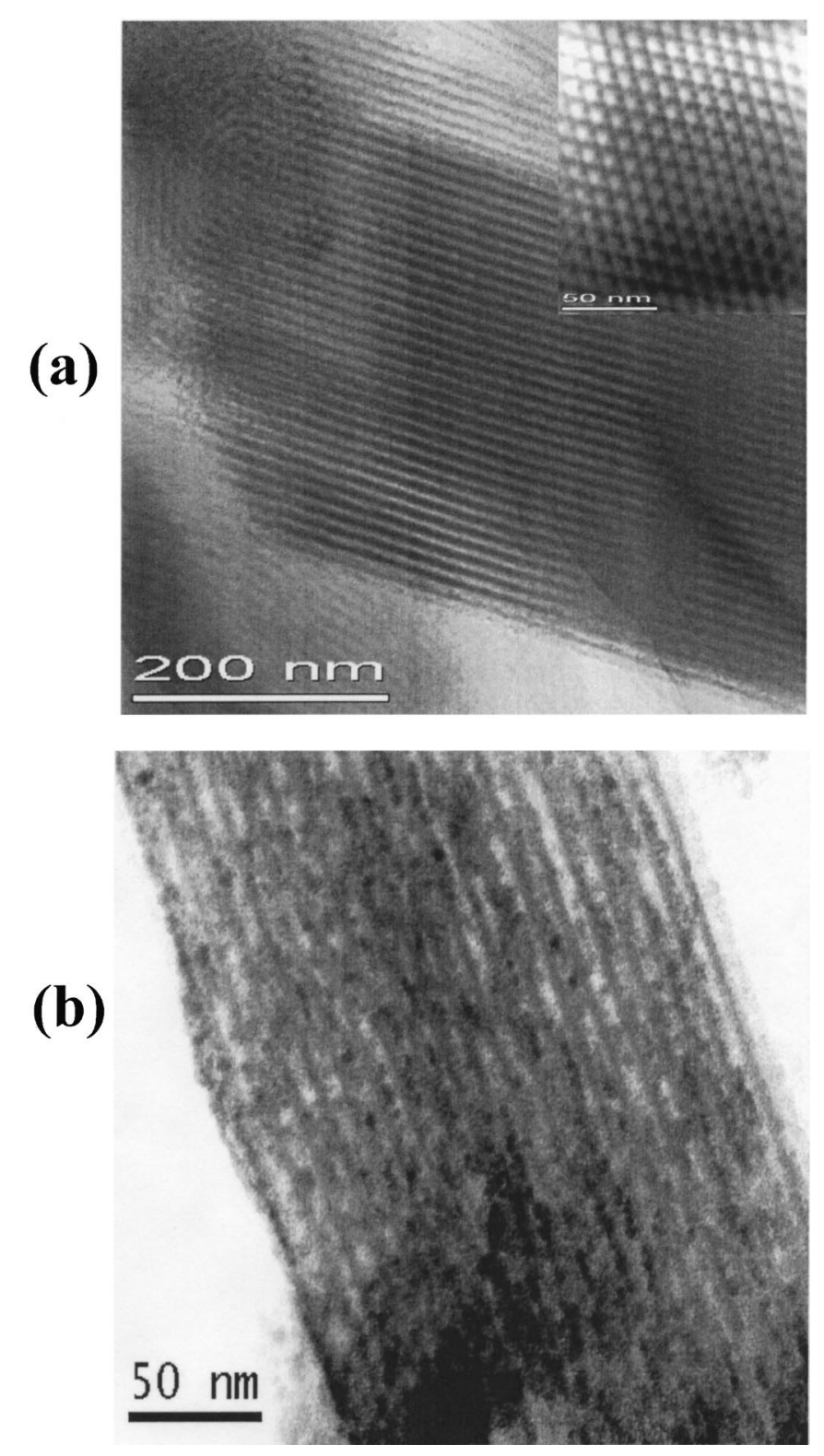

Figure 1. (a) Lateral bright-field TEM view of a mesoporous stick composed by a few hundred channels. Inset shows a front TEM view of a silica mesoporous aggregate detailing the hexagonal mesopores. (b) Tin oxide loaded SBA-15 after calcination.

at $550^{\circ} \mathrm{C}$. Their firing at higher temperature is considered to lead to a modification of the silica walls allowing a slight increase of the tin oxide particle size. The presence of the tin compound inside the channels may also help in broadening the silica pores with the thermal treatments. This deformation is observed in a more pronounced extent on MCM-41 structures with thinner walls. ${ }^{3}$ The mechanism behind this channel deformation is not straightforward to determine, and more analysis focused on the interaction of the host-support system should be carried out. For the highest calcination temperature, a compressive stress of the tin oxide structure, which is not obtained after calcination at lower temperatures, is detected on the XRD spectra (Fig. 3a). No preferential growth direction of the tin oxide nanoparticles inside the channels was observed.

A few small tin oxide particles are observed to lay outside the channels. However, no large crystallites are noticed, probably due to the limited amount of precursor tin compound laying outside the mesopores. A large percentage of the template area is attributed to the inner surface of the channels (roughly calculated to be more than 

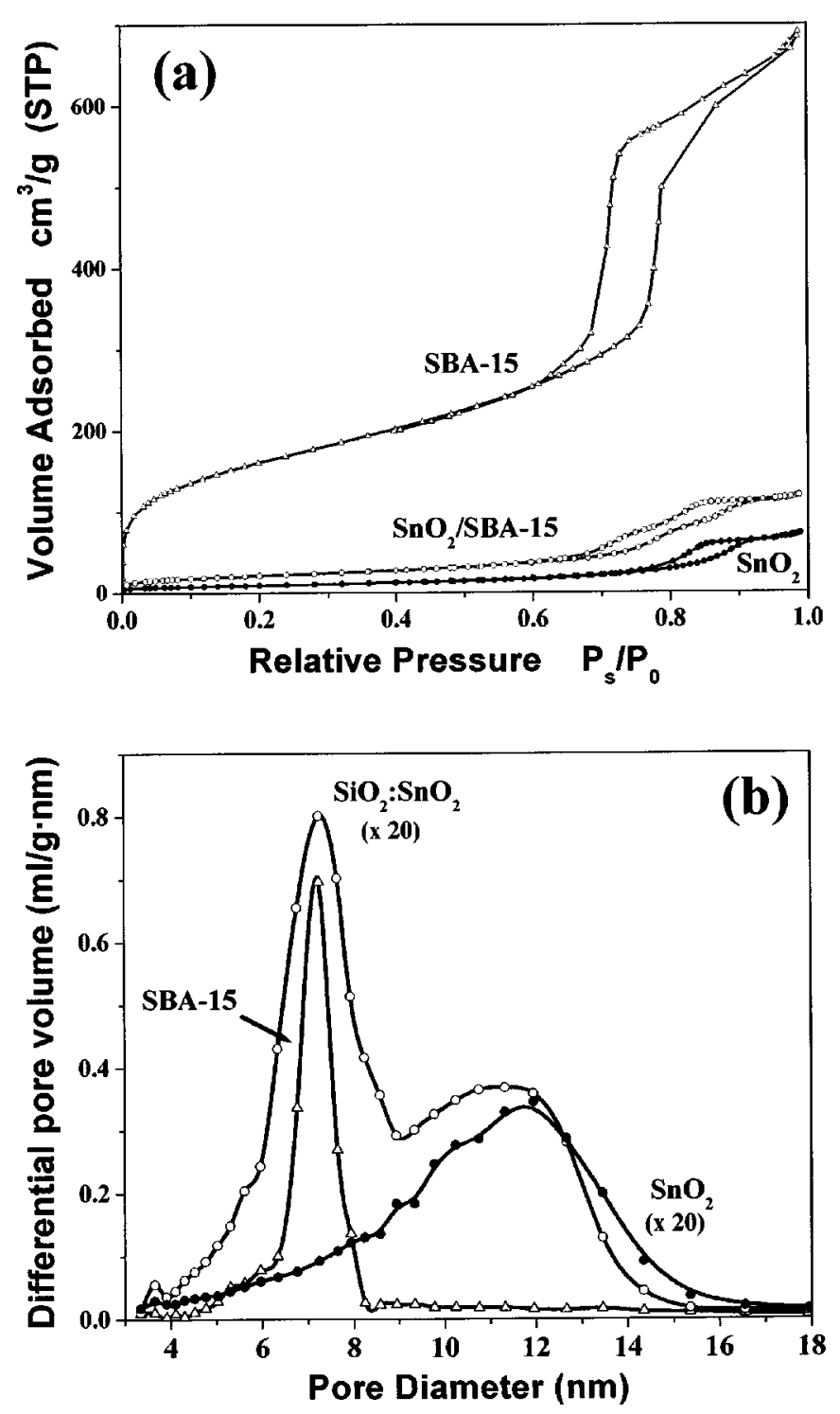

Figure 2. (a) Nitrogen sorption isotherms (77 K) and (b) BJH pore size distribution deduced from the desorption branch for the SBA-15 template silica, $\mathrm{SnO}_{2} / \mathrm{SBA}-15$, and $\mathrm{SnO}_{2}$ once the template structure has been eliminated.
$98 \%$, using geometrical considerations). Therefore, the relative quantity of material formed inside is much larger than that outside the channels, assuming the silica walls are the support for nitrate fixation and particle nucleation.

Complete filling of the pores becomes important to obtain sharp particle size distributions. Synthesizing the corseted material to relatively elevated temperatures is also crucial in obtaining stable enough nanostructures.

Figure 4a shows a TEM image of the finally obtained unsupported tin oxide nanoparticles. The template removal using an aqueous solution results in an agglomeration of the tiny crystallites. This may be prevented or afterward corrected using an appropriate dispersing agent. Statistical counting of the nanoparticles observed in TEM micrographs (Fig. 4b) is fitted with a Gaussian distribution. This Gaussian fit allows one to calculate a mean particle size of 7.9 $\mathrm{nm}$ for the sample calcined at $500^{\circ} \mathrm{C}$. This is in agreement with the pore size distribution when taking into account the systematic underestimation error on BJH pore volume, quantified to be about 1 $\mathrm{nm} .{ }^{11}$ Therefore, distribution width of the tin oxide nanoparticles from the TEM observation is comparable to that of the pore diameters obtained from the $\mathrm{N}_{2}$-sorption isotherm.

Raman spectra of the tin oxide materials calcined at 500, 600, and $800^{\circ} \mathrm{C}$ is shown in Fig. 3b. The spectra is dominated by the broad bands associated with surface related disorder. ${ }^{12}$ Superposed are observed the peaks associated to the cassiterite structure, which are identified when compared with those of the reference tin oxide. A slight shift of the A1g peak, centered at around $638 \mathrm{~cm}^{-1}$, can be discerned in the template synthesized materials when stabilizing to increasingly higher temperatures accounting for a small comprehension strain on the structure.

XPS spectra of the valence band region of the different synthesized materials are shown on Fig. 5. XPS binding energies are referred to the Fermi level of the semiconductor material, which is in electrical contact with the metallic holder. XPS measurements show a shift of the valence band of the template synthesized tin oxide to higher energies with respect to the precipitated and reference nanopowders. This shift is associated to a Fermi level displacement, at least in the surface of the material. It should correlate with a carrier density increase and/or a decrease of the intergrain barrier height.

Intergrain barriers play a dominant role in the electrical transport in nanocrystalline semiconductors. At the same time, surface structure and defects and the surrounding gas atmosphere dictate trapping of charge at the grain boundaries. In this way, the temperature dependence of the conductance is conditioned by the temperature ranges of reactivity and stability of the ionosorbed surface species. For a fixed surface charge composition, an Arrhenius dependence of the conductivity with the temperature is expected, accounting for the double grain barriers directing charge transport in polycrystalline
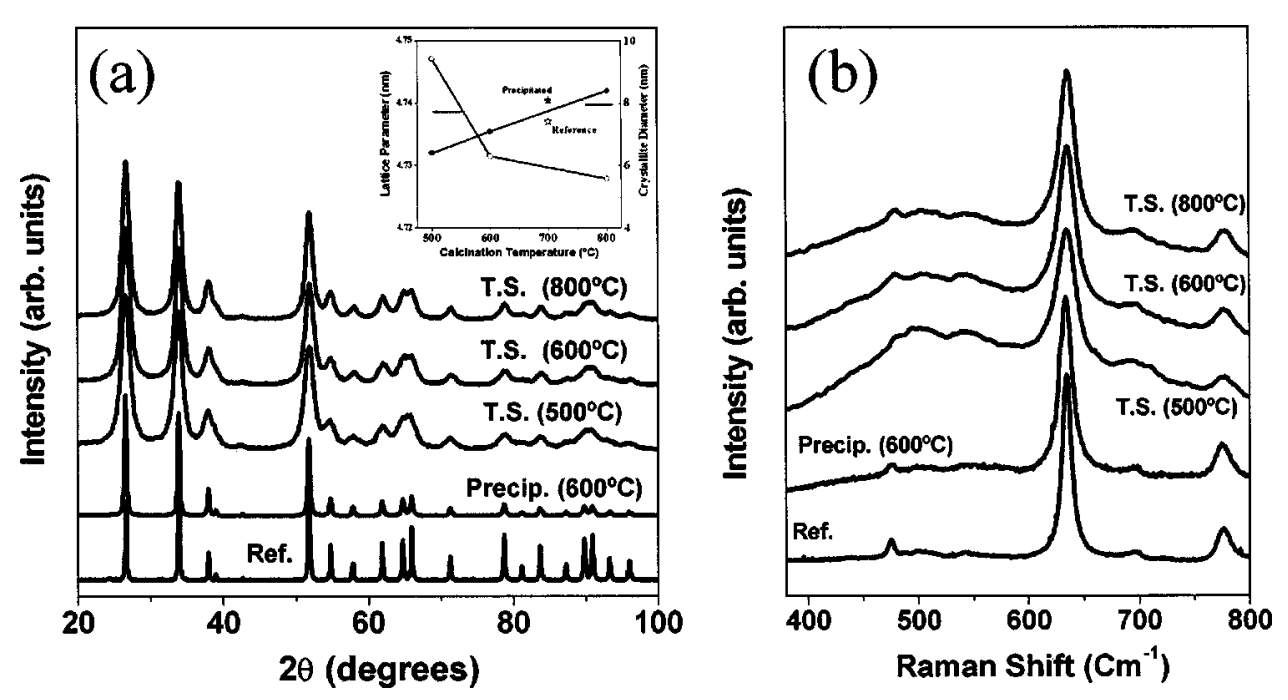

Figure 3. (a) XRD spectra and (b) Raman spectra of the reference, the precipitate, and the template synthesized tin oxide nanopowders stabilized at increasingly higher temperatures. Inset in (a) shows the particle size and the lattice parameter of the template synthesized oxides calcined at different temperatures. The lattice parameter obtained from the fitting of the (110) peak of the reference and the precipitated tin oxides are also plotted for comparison. 

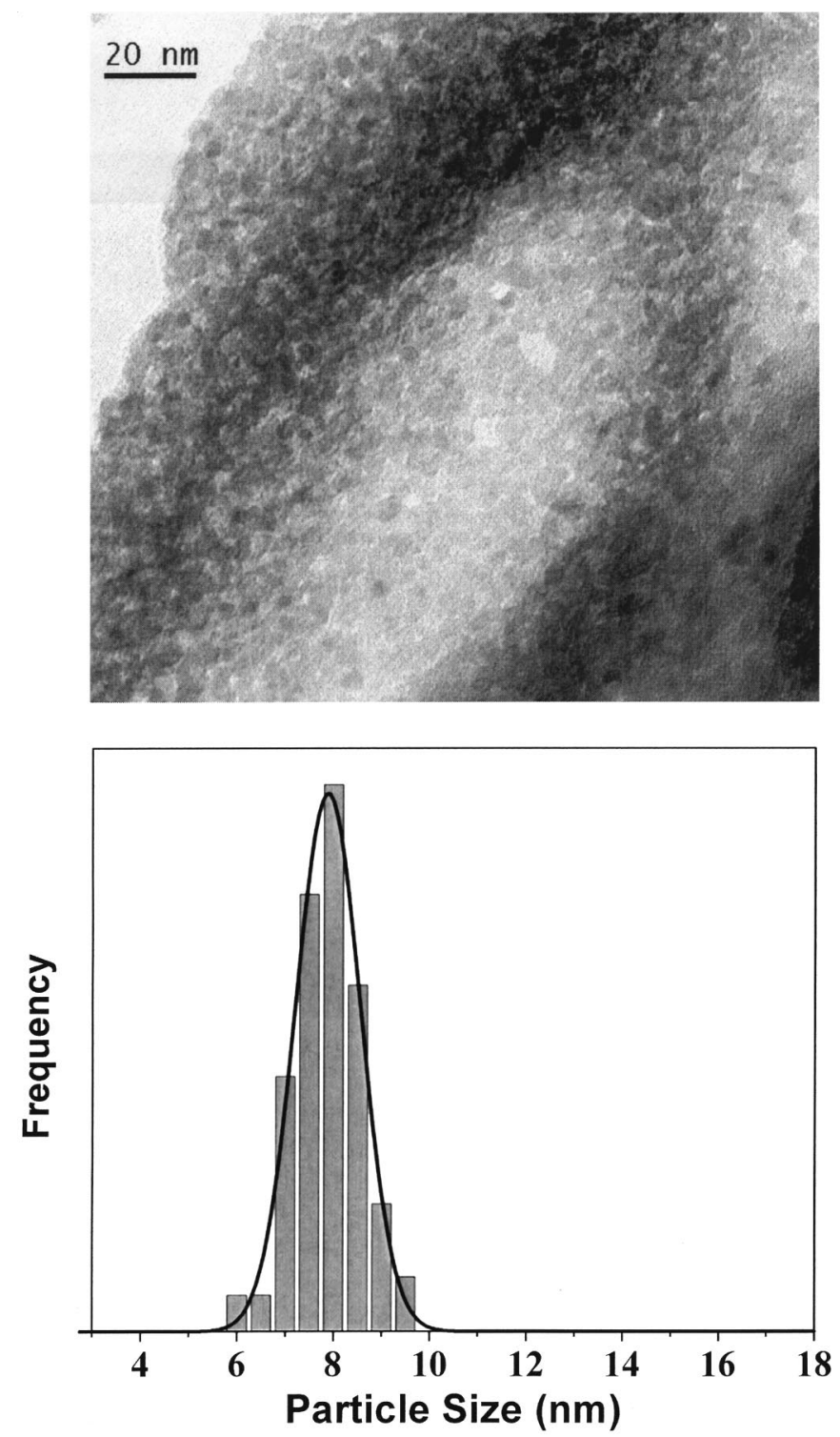

Figure 4. TEM image of the tin oxide nanoparticles once the silica template has been eliminated, and particle size distribution histogram of the tin oxide particles obtained from the TEM micrograph.

materials. ${ }^{13,14}$ With this reasoning we are considering shallow donor states completely ionized in the temperature range of operation, as is expected of tin oxide at ambient or higher temperatures. ${ }^{15}$ In a dry air atmosphere, the temperature dependence of the oxygen adsorbed species type and concentration determines main divergences of the exponential conductivity evolution with the inverse of the temperature. It also dictates the temperature ranges of some gas moleculessurface reactions.

Different tin oxides analyzed here do not have a continuous decrease of resistance with the temperature, but some steps are observed. These steps are associated to changes of the oxygen chemisorption form at the surface. Although not a large variation of the sensor conductance with respect to the reference and precipitated samples is observed, clearly different conductivity dependences with the temperature are obtained (Fig. 6). These come from provide different surface reconstruction and defects, offering different oxygen dissociation and ionosorption sites. Tiny nanoparticles are expected to offer a larger variety of sites due to surface rearrangement to yield quasi-spherical crystallites, while larger particles appear faceted. Surface morphology also determines sensitivity of the electri-

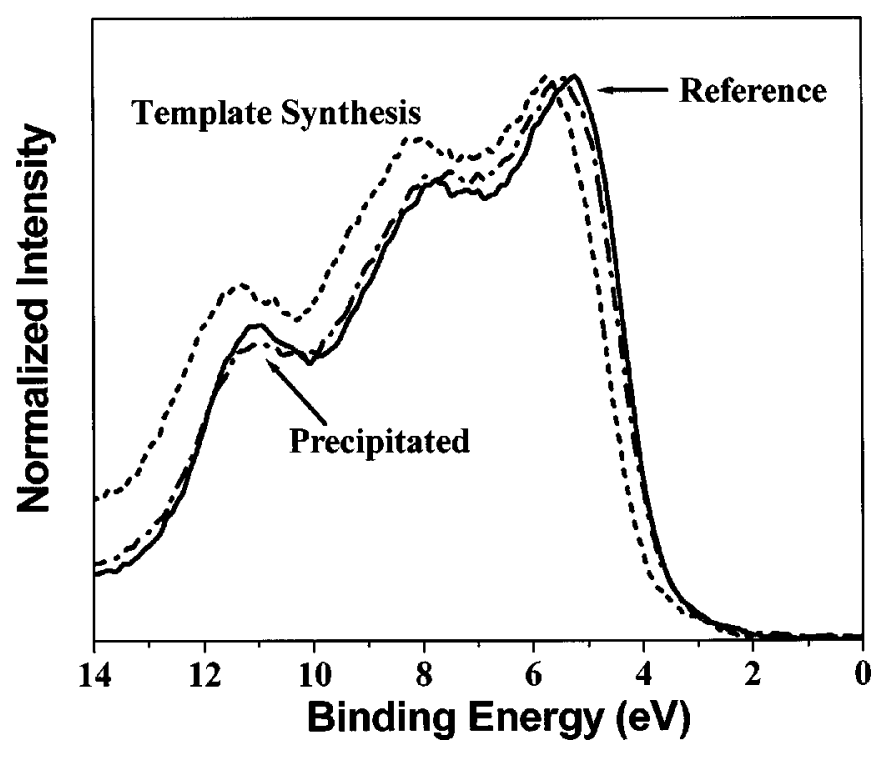

Figure 5. XPS spectra of the valence band region for the commercial, the precipitated tin oxide, and the template synthesized nanopowders calcined at $600^{\circ} \mathrm{C}$.

cal characteristics to the presence of reducing gases. In this context, when $\mathrm{CO}$ is introduced, a conductance increase, associated to a surface reduction, is obtained. This provides an increase of the surface charge density correlated with a downward band bending of the electron energy band structure at the semiconductor surface. The $\mathrm{CO}$

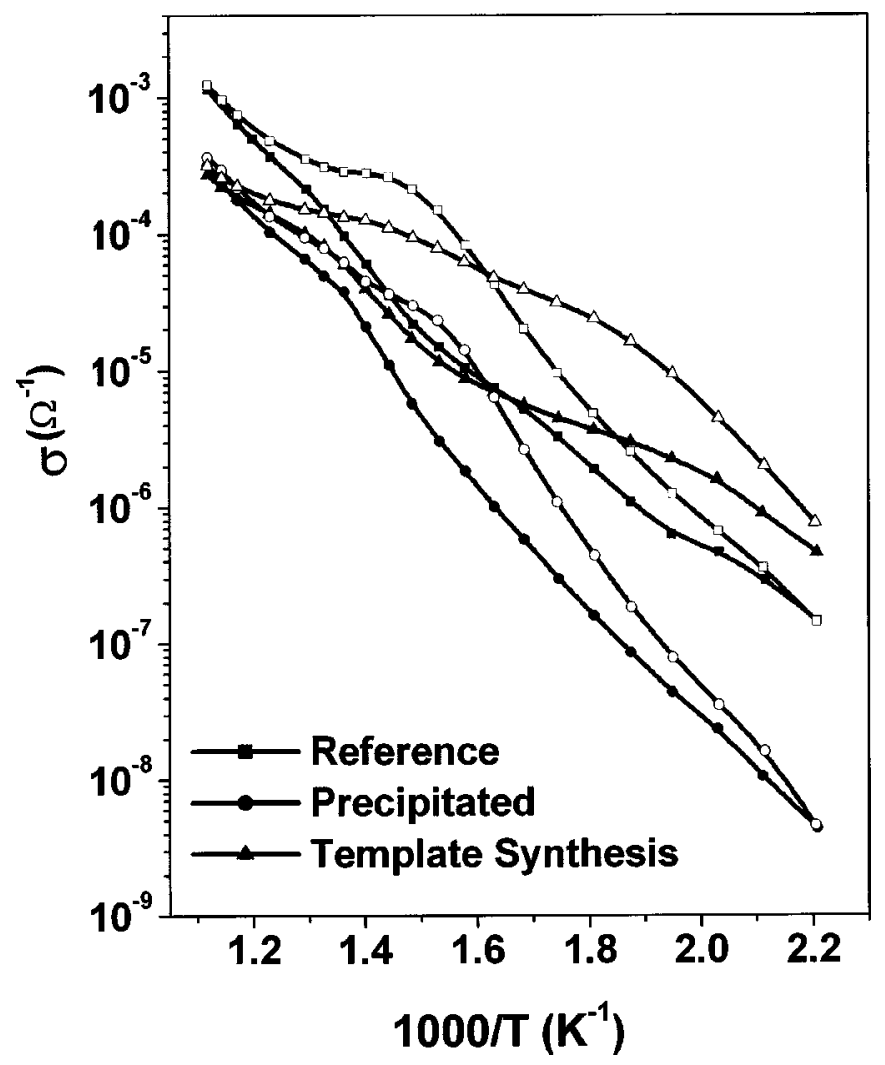

Figure 6. Conductance as a function of the inverse temperature of the sensors fabricated by printing the tin oxide materials. Filled points correspond to conductance measurements on synthetic air, while the empty points are obtained in a synthetic air atmosphere containing $100 \mathrm{ppm}$ of CO. Conductance data are obtained when increasing operating temperature. 
response of the template synthesized powders is activated at lower temperatures than those of the reference and precipitated materials. This correlates with the activation of the oxygen dissociation resulting in a much more pronounced conductivity platform for this material.

A report dealing with a more complete characterization of the surface-gas interaction properties and the related electrical responses of the template synthesized tin oxide nanoparticles is currently in progress, and will be published in the near future.

\section{Conclusions}

Tin oxide nanoparticles with controlled narrow size distributions were successfully synthesized inside silica mesoporous structures. Thus obtained tin oxide nanopowders, with particle sizes in the range between 6 and $10 \mathrm{~nm}$, showed good crystalline structure. No preferential growth direction of the crystallites inside the templates was observed. Materials were thoroughly separated from the silica structures for electrical applications. Unsupported tin oxide nanostructures were chemically and electrically characterized. The results are compared with the properties of tin oxide materials having larger particle sizes, and the observed differences in the Fermi level position and temperature dependence of the resistance associated to the presence of defects and/or reorganization of the surface structure. More information is needed at this point to determine the relation between the particle size and the surface morphology, and its detailed role on nanopowder conductance and electron energy band structure.

\section{Acknowledgments}

The authors gratefully acknowledge partial support of this project by the Georgia Institute of Technology Molecular Design
Institute under prime contract N00014-95-1-1116 from the Office of Naval Research.

Universitat de Barcelona assisted in meeting the publication costs of this article.

\section{References}

1. S. T. Chang, I. C. Leu, and M. H. Hon, Electrochem. Solid-State Lett., 5, C71 (2002); G. Pang, S. Chen, Y. Koltypin, A. Zaban, S. Feng, and A. Gedanken, Nano Lett., 1, 723 (2001); N.-L. Wu, S.-Y. Wang, and I. A. Rusakova, Science, 285, 1375 (1999).

2. A. Fukuoka, H. Araki, Y. Sakamoto, N. Sugimoto, H. Tsukada, Y. Kumai, Y. Akimoto, and M. Ichikawa, Nano Lett., 2, 793 (2002).

3. S. Besson, T. Gacoin, C. Ricolleau, C. Jacquiod, and J.-P. Boilor, Nano Lett., 2, 409 (2002).

4. G. Che, B. B. Lakshmi, E. R. Fisher, and C. R. Martin, Nature (London), 293, 346 (1998).

5. S. H. Joo, S. J. Choi, I. Oh, J. Kwak, Z. Liu, O. Terasaki, and R. Ryoo, Nature (London), 412, 169 (2001)

6. L. Chen, P. J. Klar, W. Heimbrodt, F. Brieler, and M. Fröba, Appl. Phys. Lett., 76, 3531 (2000).

7. C. R. Martin, Science, 266, 1961 (1994); C. R. Martin, Chem. Mater, 8, 1739 (1996).

8. J. Sauer, F. Marlow, B. Spliethoff, and F. Schüth, Chem. Mater, 14, 217 (2002)

9. D. Zhao, J. Feng, Q. Huo, N. Melosh, G. H. Fredrickson, B. F. Chmelka, and G. D. Stucky, Science, 279, 548 (1998); D. Zhao, Q. Huo, J. Feng, B. F. Chmelka, and G. D. Stucky, J. Am. Chem. Soc., 120, 6024 (1998).

10. B. Marler, U. Oberhagemann, S. Vortmann, and H. Gies, Microporous Mater, 6, 375 (1996); W. Hammond, E. Prouzet, S. D. Mahanti, and T. J. Pinnavaia, Microporous Mesoporous Mater., 27, 19 (1999).

11. W. W. Lukens, Jr., P. Schmidt-Winkel, D. Zhao, J. Feng, and G. D. Stucky, Langmuir, 15, 5403 (1999).

12. N.-L. Wu, L.-F. Wu, I. A. Rusakova, A. Hamed, and A. P. Litvinchuk, J. Am. Ceram. Soc., 82, 67 (1999)

13. F. Greuter and G. Blatter, Semicond. Sci. Technol., 5, 111 (1990).

14. G. E. Pike, Phys. Rev. B, 30, 795 (1984).

15. S. Samson and C. G. Fonstad, J. Appl. Phys., 44, 4618 (1973) 